\title{
A Dynamical Mechanism for Quark Mixing and Neutrino Oscillations
}

\author{
José BORDES \\ jose.m.bordes@ uv.es \\ Dept. Fisica Teorica, Univ. de Valencia, \\ c. Dr. Moliner 50, E-46100 Burjassot (Valencia), Spain \\ CHAN Hong-Mo \\ chanhm@v2.rl.ac.uk \\ Rutherford Appleton Laboratory, \\ Chilton, Didcot, Oxon OX11 0QX, United Kingdom \\ TSOU Sheung Tsun \\ tsou@ maths.ox.ac.uk \\ Mathematical Institute, University of Oxford, \\ 24-29 St. Giles', Oxford OX1 3LB, United Kingdom
}

\begin{abstract}
We show that assuming fermion generations to be given by a gauge symmetry plus a certain Higgs mechanism for its breaking, the known empirical features of quark and lepton mixing can be largely explained, including in particular the fact that the mixing (CKM) matrix element $U_{\mu 3}$ responsible for the muon anomaly in atmospheric neutrinos is near maximal and much larger than their quark counterparts $V_{c b}$ and $V_{t s}$, while the corner elements for both quarks $\left(V_{u b}, V_{t d}\right)$ and leptons $\left(U_{e 3}\right)$ are all very small. The mechanism also gives automatically a hierarchical fermion mass spectrum which is intimately related to the mixing pattern.
\end{abstract}


The quark mixing pattern as measured by the Cabibbo-Kobayashi-Moskawa (CKM) matrix is now quite well-known. The latest databook [1] gives the absolute values of the matrix elements as:

$$
\begin{aligned}
& \left(\begin{array}{lll}
\left|V_{u d}\right| & \left|V_{u s}\right| & \left|V_{u b}\right| \\
\left|V_{c d}\right| & \left|V_{c s}\right| & \left|V_{c b}\right| \\
\left|V_{t d}\right| & \left|V_{t s}\right| & \left|V_{t b}\right|
\end{array}\right)= \\
& \left(\begin{array}{lll}
0.9745-0.9760 & 0.217-0.224 & 0.0018-0.0045 \\
0.217-0.224 & 0.9737-0.9753 & 0.036-0.042 \\
0.004-0.013 & 0.035-0.042 & 0.9991-0.9994
\end{array}\right)
\end{aligned}
$$

Information on the corresponding matrix for leptons is beginning also to emerge from recent experiments on neutrino oscillations. In particular, the result from atmospheric neutrinos [2, 3, 4, 5] shows that the mixing angle between $\nu_{\mu}$ and the heaviest $\nu_{3}$ state is near maximal, while the absence of oscillation effects in some reactor experiments, in particular CHOOZ [6], implies that the mixing of $\nu_{e}$ to the same heaviest state $\nu_{3}$ is rather small. From solar neutrino data, the picture is not yet entirely clear. Of the 3 traditional solutions, namely (i) the small angle MSW, (ii) the large angle MSW, and (iii) the long wave-length (or vacuum, or just-so) oscillation (LWO), both (i) and (ii) are under pressure from the latest Superkamiokande data on daynight variation and flux [7], which seem to have a slight preference for (iii), but the situation is still far from settled. On can conclude at present only that the angle between $\nu_{e}$ and the second heaviest state $\nu_{2}$ is either quite small (i) or again near maximal (ii)-(iii). As a result, a CKM matrix is suggested roughly of the form:

$$
\left(\begin{array}{ccc}
\left|U_{e 1}\right| & \left|U_{e 2}\right| & \left|U_{e 3}\right| \\
\left|U_{\mu 1}\right| & \left|U_{\mu 2}\right| & \left|U_{\mu 3}\right| \\
\left|U_{\tau 1}\right| & \left|U_{\tau 2}\right| & \left|U_{\tau 3}\right|
\end{array}\right)=\left(\begin{array}{ccc}
\star & 0.4-0.7 & 0.0-0.15 \\
\star & \star & 0.56-0.83 \\
\star & \star & \star
\end{array}\right)
$$

where, for reasons which will be apparent later, we have inserted for $U_{e 2}$ the value suggested by the LWO solution (iii). If CP-violations are ignored, the elements denoted by $\star$ are obtainable by unitarity from the others.

In these mixing matrices, one notices some very outstanding features:

(a) The off-diagonal elements in the quark CKM matrix are all small or very small; 
(b) The corner elements in both the quark and lepton matrices are all very much smaller than the others;

(c) The $U_{\mu 3}$ element in the lepton matrix is much (about a factor 20) larger than its quark counterparts, namely $V_{c b}$ and $V_{t s}$.

These features, together with the actual values that the elements take, cry out urgently for a theoretical explanation.

What we wish to show in this paper is that all the above features together with the hierarchical fermion mass spectrum can very simply be explained and even semi-quantitatively calculated in terms of a few parameters if one assumes generation to be an $S U(3)$ gauge symmetry spontaneously broken in a particular manner. This observation is abstracted from a recently proposed scheme we called the Dualized Standard Model (DSM) [8, 9] based on a nonabelian generalization of electric-magnetic duality [10]. Here we shall do the following. First, we shall distill and simplify the arguments to such an extent as to make the mechanism, we hope, completely transparent. Secondly, we shall make clear that the main mechanism is independent of the concept of duality, thus freeing it from our own theoretical bias, so that if one prefers (which we ourselves do not for reasons to be given later) one can obtain similar results by grafting the proposed mechanism on to some different, not necessarily dual, scheme. Thirdly, we shall present a new, more systematic, fit together with a more detailed comparison with experiment using the latest data while making some points of detail not noted before.

The idea that generation originates from a (spontaneously broken) 'horizontal' gauge symmetry is not new. The empirical fact that fermions seem to occur in 3 and only 3 generations suggests $S U(3)$. In analogy to the electroweak theory, we then propose to assign left-handed fermions to the fundamental triplet representation and right-handed fermions to singlets. For breaking the symmetry, a possibility is to introduce $3 S U(3)$ triplets of Higgs fields, say $\phi^{(a)}, a=1,2,3$, with linearly independent, say mutually orthogonal, vacuum values, namely that $\bar{\phi}^{(a)} \phi^{(b)}=0, a \neq b$ at vacuum. Furthermore, we stipulate that the 3 Higgs triplets be 'indistinguishable' so that the action has to be symmetric under their permutations, although the vacuum need not be thus symmetric.'

\footnotetext{
${ }^{1}$ In the DSM, these proposals are given some raison d'être since there the $\phi$ 's are related to frame vectors in $U(3)$, but one need take no account of that if one so prefers.
} 
A possible potential for these Higgs fields is then:

$$
V[\phi]=-\mu \sum_{(a)}\left|\phi^{(a)}\right|^{2}+\lambda\left\{\sum_{(a)}\left|\phi^{(a)}\right|^{2}\right\}^{2}+\kappa \sum_{(a) \neq(b)}\left|\bar{\phi}^{(a)} \cdot \phi^{(b)}\right|^{2},
$$

for which a general vacuum can be expressed as:

$$
\phi^{(1)}=\zeta\left(\begin{array}{l}
x \\
0 \\
0
\end{array}\right) ; \phi^{(2)}=\zeta\left(\begin{array}{l}
0 \\
y \\
0
\end{array}\right) ; \phi^{(3)}=\zeta\left(\begin{array}{l}
0 \\
0 \\
z
\end{array}\right),
$$

with

$$
\zeta=\sqrt{\mu / 2 \lambda}
$$

and $x, y, z$ all real and positive, satisfying:

$$
x^{2}+y^{2}+z^{2}=1 .
$$

Such a vacuum breaks the permutation symmetry of the $\phi$ 's, and also the $S U(3)$ gauge symmetry completely. As a result, all the vector gauge bosons in the theory acquire a mass, eating up all but 9 of the Higgs modes?

Next, given the above assignments of $S U(3)$ representations to the leftand right-handed fermions, the Yukawa couplings take the form:

$$
\sum_{(a)[b]} Y_{[b]} \bar{\psi}_{L}^{a} \phi_{a}^{(a)} \psi_{R}^{[b]}
$$

which is symmetric under permutations of $\phi^{(a)}$ as required. As a result, the tree-level mass matrix for each of the 4 fermion-types $T$ (i.e. whether $U$ - or $D$-type quarks, or charged leptons (L) or neutrinos $(\mathrm{N}))$ is of the following factorized form:

$$
m \propto\left(\begin{array}{l}
x \\
y \\
z
\end{array}\right)(a, b, c)
$$

with $a, b, c$ being the Yukawa couplings $Y_{[b]}$. Of more relevance to the mass spectrum is the matrix $\mathrm{mm}^{\dagger}$ which takes the form:

$$
\sqrt{m m^{\dagger}}=m_{T}\left(\begin{array}{l}
x \\
y \\
z
\end{array}\right)(x, y, z) .
$$

\footnotetext{
${ }^{2}$ The $\phi$ 's in fact break a larger $U(3)$ symmetry, giving thus 9 massive vector bosons.
} 
This is of rank 1 , having only one nonzero eigenvalue with eigenvector $(x, y, z)$ the components of which, being Higgs vev's, are independent of the fermiontype $T$. Hence we have already at tree-level (i) that the fermion mass spectrum is 'hierarchical' with one generation much heavier than the other two, (ii) that the CKM matrix giving the relative orientation between the eigenvectors of the up- and down-type fermions is the identity matrix. Both of these conditions give sensible zero-order approximations, at least for quarks, to the experimental data.

Consider next 1-loop corrections. It is not hard to see that the corrected fermion mass matrix $m^{\prime}$ will remain in a factorized form. The reason is that only those loops involving the generation-changing gauge and Higgs bosons can affect the factorization, and of these the gauge bosons couple only to the left-handed fermions while the Higgs bosons have couplings which are themselves factorizable. Indeed, it appears that the factorized mass matrix will survive to all orders in perturbation. As a result, we have:

$$
\sqrt{m^{\prime} m^{\prime \dagger}}=m_{T}^{\prime}\left(\begin{array}{c}
x^{\prime} \\
y^{\prime} \\
z^{\prime}
\end{array}\right)\left(x^{\prime}, y^{\prime}, z^{\prime}\right),
$$

where the corrected vector $\left(x^{\prime}, y^{\prime}, z^{\prime}\right)$ depends both on the fermion-type and on the energy scale. At the 1-loop level, the vector $\left(x^{\prime}, y^{\prime}, z^{\prime}\right)$ remains real so that there is no $C P$-violation at this level.

The scale-dependence of $m^{\prime}$ above is a special case of a mass matrix which rotates with the energy scale. In itself, this is not unusual since already in the standard formulation of the Standard Model, such a rotation of the fermion mass matrix will result in the renormalization group equation from a nondiagonal CKM matrix [11], although the effect there is small and therefore usually neglected. When the effect of the rotation is appreciable, as it can be in our present case, then care has to be exercised in its physical interpretation. When the mass matrix does not rotate with scale, as in QCD where the scaledependence induced by gluonic corrections appears as an overall flavourindependent factor, there is of course no difficulty in identifying the masses and state vectors of the physical states. The matrix can be diagonalized at any scale giving a set of eigenvectors independent of the scale although the eigenvalues themselves will in general be scale-dependent. These eigenvectors can then be taken unambiguously as the state vectors of the physical states while the mass of each physical state can be defined as the running eigenvalue 
$m_{i}(\mu)$ corresponding to the state $i$ when taken at the scale equal to its value, namely as the solution to the equations $m_{i}(\mu)=\mu$. However, if the mass matrix rotates with scale, then its eigenvectors are also scale-dependent and it becomes unclear how the physical state vectors are to be defined. One may be tempted to define the eigenvector for the value $m_{i}$ at the scale $\mu$ satisfying the equation $m_{i}(\mu)=\mu$ as the state vector for the physical state $i$, but the state vectors defined in this way will not be mutually orthogonal, thus contradicting the ansatz that they represent physically independent quantum states.

The solution we propose to adopt in this paper, which is in fact the only one we can think of, is as follows. We run the mass matrix $m$ down in scale until we have for its highest eigenvalue $m_{3}$ a solution to the equation $m_{3}(\mu)=\mu$. This value at this scale we define as the mass $m_{3}$, and the corresponding eigenvector the state vector $\mathbf{v}_{\mathbf{3}}$ of the heaviest generation. Below that energy, the state 3 no longer exists as a physical state, and only the two lighter generations survive, the state vectors of which have to be orthogonal to $\mathbf{v}_{\mathbf{3}}$. We define therefore the mass matrix at energies below $m_{3}$ as the $2 \times 2$ submatrix $\hat{m}$ of $m$ in the subspace orthogonal to $\mathbf{v}_{\mathbf{3}}$. To find now the mass and state vector for generation 2 we follow with $\hat{m}$ the same procedure as for 3 with $m$ and run $\hat{m}$ down in scale until we find a solution to the equation $\hat{m}_{2}(\mu)=\mu$, which value we call the mass $m_{2}$ and the corresponding eigenvector at that scale the state vector $\mathbf{v}_{\mathbf{2}}$ of the generation 2 . The state vector of the lightest generation 1 is now also defined, as the vector orthogonal to both $\mathbf{v}_{\mathbf{3}}$ and $\mathbf{v}_{\mathbf{2}}$, while the mass of 1 will obtain by repeating the above procedure, namely by running down in scale the expectation value $\left\langle\mathbf{v}_{\mathbf{1}}|m| \mathbf{v}_{\mathbf{1}}\right\rangle$ until its value equals the scale. In this way, each mass is evaluated at its own appropriate scale while the physical state vectors of the 3 generations are all mutually orthogonal, as they should be.

Applying the above procedure to the factorized mass matrix $m^{\prime}$ in (10), one sees that for the heaviest generation fermion of type $T$, the mass $m_{3}$ is $m_{T}^{\prime}$ and the state vector $\mathbf{v}_{\mathbf{3}}$ is $\left(x^{\prime}, y^{\prime}, z^{\prime}\right)$, both taken at the scale $\mu$ satisfying the condition $m_{T}^{\prime}(\mu)=\mu$. At that scale, the subspace orthogonal to $\mathbf{v}_{\mathbf{3}}$ has zero mass eigenvalues, and it is as yet unclear which vector in it should correspond to the second and which the lightest generation. However, as the scale lowers further, the vector $\left(x^{\prime}, y^{\prime}, z^{\prime}\right)$ rotates to a different direction giving nonzero components in the orthogonal subspace and hence a nonzero eigenvalue to $\hat{m}^{\prime}$. One can then define this nonzero value as $\hat{m}_{2}^{\prime}(\mu)$ and pro- 
cede as above to determine the (nonzero) mass $m_{2}$ and state vector $\mathbf{v}_{\mathbf{2}}$ of the second generation. At the same time one determines the state vector $\mathbf{v}_{\mathbf{1}}$ of the lightest generation. The triad of state vectors so determined for the 3 generations are as shown in Figure 1. The mass of the lightest generation can also be found by running the scale down further. As a result, all 3 generations will acquire finite masses by this 'leakage' mechanism, but the mass spectrum will be hierarchical, meaning that $m_{3} \gg m_{2} \gg m_{1}$, qualitatively as experimentally observed. Further, since a triad of state vectors for the 3 generations have now been defined for each fermion-type, CKM matrix elements can be evaluated as the direction cosines between the state vectors of the various up- and down-type fermions. And since the loop corrections are in general different for up- and down-types, the resulting matrix will be nondiagonal giving nonzero mixing.

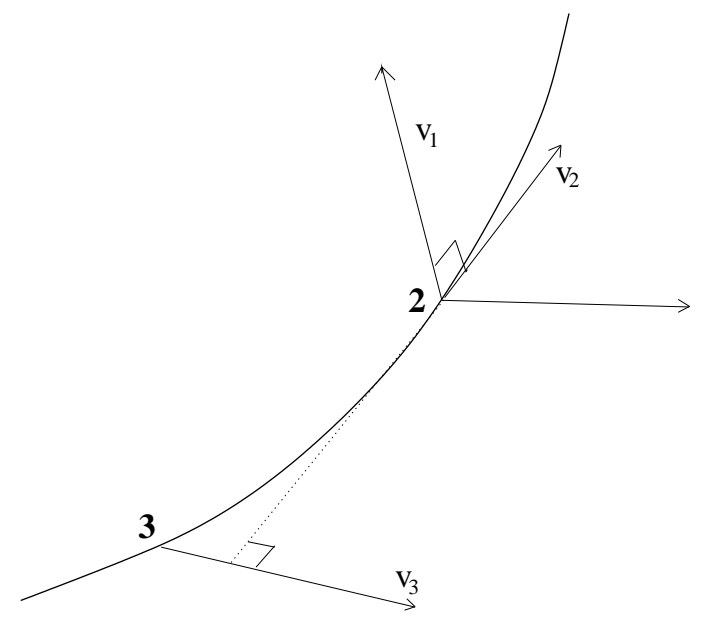

Figure 1: The triad of state vectors for the 3 generations of fermions.

One sees therefore that in the present framework with a factorized mass matrix, nearly all the information on fermion mixing and much of that on the fermion mass spectrum are encoded in a single 3 -vector $\left(x^{\prime}, y^{\prime}, z^{\prime}\right)$ in generation space, one for each fermion-type. This vector rotates with the energy scale and as the scale changes, it traces out a trajectory on the unit sphere. By studying the shape of these trajectories and the speed at which $\left(x^{\prime}, y^{\prime}, z^{\prime}\right)$ moves along them, one will be able to deduce properties of the CKM matrix and the fermion mass spectrum. 
Let us then examine in more detail how loop corrections affect the vector $\left(x^{\prime}, y^{\prime}, z^{\prime}\right)$. As already noted, only those loop diagrams involving generationchanging bosons can rotate the vector $\left(x^{\prime}, y^{\prime}, z^{\prime}\right)$. A closer examination then reveals [12] that of the various 1-loop diagrams, only 3 give rotations, namely those in Figure 2, where a full line denotes a fermion, a wriggly line a generation-changing gauge boson and a dashed line a generation-changing Higgs boson of the type $\phi^{(a)}$ detailed above. Of these remaining diagrams, Figures 2(a) and (b) give rotations of order $m^{2} / \zeta_{0}^{2}$ (where $\zeta_{0}$ is the smallest Higgs vev) and are constrained by experiment to be negligible for the following reason. As noted before, in breaking the generation $S U(3)$ symmetry, the corresponding gauge bosons all acquire masses of order or higher than $g \zeta_{0}, g$ being the gauge coupling. The exchange of these bosons will lead to flavour-changing neutral current (FCNC) effects at low energies of the order $g^{2} /\left(g \zeta_{0}\right)^{2} \sim 1 / \zeta_{0}^{2}$. Present experimental bounds on FCNC effects, such as an anomalous $K_{L}-K_{S}$ mass difference, will thus lead to very stringent lower bounds on the value of $\zeta_{0}$, which is currently of the order $100 \mathrm{TeV}$ [13]. Hence the rotation due to Figures (a) and (b), even for the top quark of mass 180 $\mathrm{GeV}$, is only of order $10^{-6}$ and therefore entirely negligible. There remains then only the Higgs loop diagram (c) to be considered.

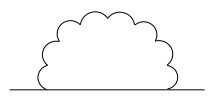

(a)

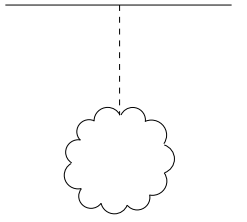

(b)

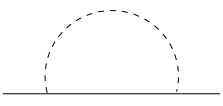

(c)

Figure 2: One loop diagrams rotating the fermion mass matrix.

The rotation from the diagram (c) has been evaluated [12] and gives:

$$
\frac{d}{d\left(\ln \mu^{2}\right)}\left(\begin{array}{c}
x^{\prime} \\
y^{\prime} \\
z^{\prime}
\end{array}\right)=\frac{5}{64 \pi^{2}} \rho^{2}\left(\begin{array}{c}
\tilde{x}_{1}^{\prime} \\
\tilde{y}_{1}^{\prime} \\
\tilde{z}_{1}^{\prime}
\end{array}\right),
$$

with

$$
\tilde{x}_{1}^{\prime}=\frac{x^{\prime}\left(x^{\prime 2}-y^{\prime 2}\right)}{x^{\prime 2}+y^{\prime 2}}+\frac{x^{\prime}\left(x^{\prime 2}-z^{\prime 2}\right)}{x^{\prime 2}+z^{\prime 2}}, \quad \text { cyclic, }
$$


and $\rho^{2}=|a|^{2}+|b|^{2}+|c|^{2}$ being the Yukawa coupling strength. By iterating this formula, one can compute the trajectory traced out by the vector $\left(x^{\prime}, y^{\prime}, z^{\prime}\right)$ given any initial value.

The choice of an initial value of the vector $\left(x^{\prime}, y^{\prime}, z^{\prime}\right)$, which fixes the trajectory it is on, depends in principle on the original vev's $x, y, z$ of the Higgs fields, the masses of the Higgs bosons, and also the Yukawa coupling strength $\rho$, the last of which depends in turn on the fermion-type. One can thus attempt a global fit to the empirical CKM matrix and fermion mass spectrum with these quantities as parameters. This was the approach adopted in 12 and a good fit has been obtained. In this paper, however, we shall consider only a particular solution suggested by the fit in [12 which we believe may have a deeper meaning than is as yet fully understood, namely when the Yukawa coupling strength $\rho$ is the same for all fermion-types. . $^{3}$ In this case, the vector $\left(x^{\prime}, y^{\prime}, z^{\prime}\right)$ runs on the same trajectory with the same speed for all fermion-types which differ thus only in the positions that their physical states occupy on the common trajectory. This simplifies the problem considerably and renders the mechanism very transparent since the whole set-up now depends on only 3 (real) parameters, namely the common Yukawa coupling strength $\rho$ and a common (normalized) initial vector $\left(x_{I}, y_{I}, z_{I}\right)$ at some (high) arbitrary scale. With these, as we shall see, one can already explain semi-quantitatively nearly all the features of quark and lepton mixing noted above, while making as well some rough estimates for the lower generation fermion masses given the masses of the heaviest generation.

Before we proceed to a formal fit of the data with the 3 remaining parameters, let us first examine the problem qualitatively to try to anticipate the form that such a fit will take. From (11) and (12), one sees that $(1,0,0)$ and $\frac{1}{\sqrt{3}}(1,1,1)$ are both fixed points on the trajectory, and that when going down in energy scale, the vector $\left(x^{\prime}, y^{\prime}, z^{\prime}\right)$ runs away from $(1,0,0)$ towards $\frac{1}{\sqrt{3}}(1,1,1)$. It will run, of course, faster in the middle than near the fixed points, at a speed the actual value of which depends on the Yukawa coupling strength $\rho$.

Consider first the fermion masses of the two highest generations, where one recalls that in the present set-up masses of the second generation arise

\footnotetext{
${ }^{3}$ Notice that the normalization of the mass matrix is not calculable perturbatively if the coupling is large as in the DSM scheme, and has thus to be regarded in general as a different parameter from the Yukawa coupling $\rho$ in the present framework.
} 
only by 'leakage' from the highest generation. It follows then from the observation in the above paragraph that those situated near the fixed points will acquire proportionately smaller masses from 'leakage' since the running is there less efficient. Given now the empirical pattern that $m_{c} / m_{t}<m_{s} / m_{b}<$ $m_{\mu} / m_{\tau}$, while $m_{t}>m_{b}>m_{\tau}$, namely the heavier the mass the smaller the 'leakage', it seems advisable in attempting a fit to place $m_{t}$ fairly close to the high energy fixed point $(1,0,0)$, so that $m_{b}$ and $m_{\tau}$ being lower in mass and hence further away from the fixed point will 'leak' more of their masses into their second generation states. The resulting arrangement for the 2 highest generation states of the 3 fermion-types $U, D, L$ would then roughly be as shown in Figure 3 .

For neutrinos $N$, the consideration is a little more complicated. What enter in the 'leakage' argument of Figure 3 are the Dirac masses $M_{\nu_{i}}$, but neutrinos can also have a Majorana mass $B$ f. The physical masses $m_{\nu_{i}}$ for the 3 generations of neutrinos are given by the see-saw mechanism as $M_{\nu_{i}}^{2} / B$. Experimentally, if neutrino masses are assumed to be hierarchical, as they must be in the present set-up, the data on atmospheric neutrinos [2, 3, 4] give a (physical) mass to the heaviest neutrino $\nu_{3}$ of order $m_{\nu_{3}}^{2} \sim 10^{-3}-10^{-2} \mathrm{eV}^{2}$. For the second generation neutrino $\nu_{2}$, solar neutrino data suggest a (physical) mass of either $m_{\nu_{2}}^{2} \sim 10^{-5} \mathrm{eV}^{2}$ if one takes the MSW solution [14], or $m_{\nu_{2}}^{2} \sim 10^{-10} \mathrm{eV}^{2}$ if one takes the LWO solution [15]. In the MSW case, one obtains then $M_{\nu_{2}} / M_{\nu_{3}} \sim 0.18-0.31$, while in the LWO case $M_{\nu_{2}} / M_{\nu_{3}} \sim 0.010-0.018$. This ratio for the MSW case is much bigger than the corresponding figures for the other 3 fermion-types $U, D, L$, which in the present set-up means also bigger 'leakage efficiency'. Indeed, the 'leakage' required by the MSW solution is so big that one is easily convinced by a few trial calculations that it cannot be accommodated here even if $\rho$ is allowed to take a very different value from the other 3 fermion-types. On the other hand, the 'leakage efficiency' required by the LWO solution, which is only somewhat bigger than that of the $U$-type quarks, can be readily accommodated. Since the Dirac masses of neutrinos (dependent on $B$ ) are empirically unknown, the heaviest state $\nu_{3}$ can in principle be assigned any location on the trajectory so long as it gives a correct 'leakage efficiency' to reproduce

\footnotetext{
${ }^{4}$ In order for the 'leakage' mechanism to work for neutrinos as for the other fermiontypes, they have also to be Dirac fermions with their left-handed components forming a triplet of the horizontal $S U(3)$ symmetry and their right-handed components $S U(3)$ singlets having a common Majorana mass.
} 
the mass ratio $M_{\nu_{2}} / M_{\nu_{3}}$. One obvious possibility is to locate $\nu_{3}$ close to $t$ but this will make the lepton CKM matrix very similar to that of the quarks. A much more interesting possibility is to place $\nu_{3}$ far down the same trajectory, as illustrated in Figure 3, where since the vector $\left(x^{\prime}, y^{\prime}, z^{\prime}\right)$ is now pressing against the low energy fixed point $\frac{1}{\sqrt{3}}(1,1,1)$ the 'leakage efficiency' is again reduced, say compared to $D$-type quarks and charged leptons, as required. We choose to consider this second possibility.

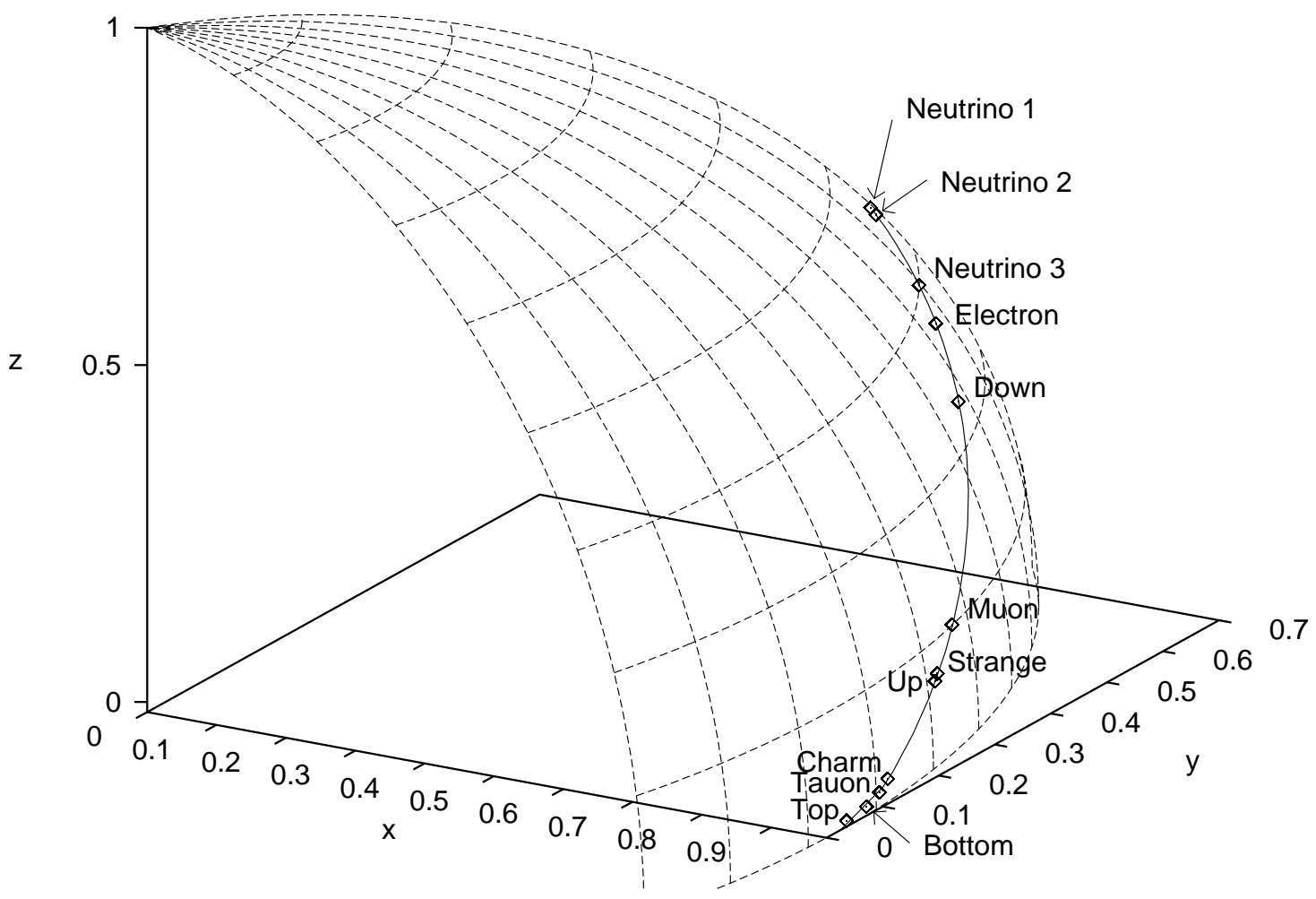

Figure 3: Trajectory traced out by the vector $\left(x^{\prime}, y^{\prime}, z^{\prime}\right)$.

As explained above, giving the locations on the trajectory of the 2 highest generation states in the present set-up also fixes the triad of state vectors of all 3 generations. It is then a simple matter to evaluate the CKM matrix the elements of which are just the direction cosines between the triads of the 
$U$ - and $D$-type quarks, or else for leptons, between the triads of the charged leptons $L$ and the neutrinos $N$. Given that in Figure 3, the quarks are much closer in location than the leptons, so also will be their triads in orientation. It follows then immediately (a) that the CKM matrix is much closer to the identity for quarks than for leptons, a qualitative fact clearly borne out by a comparison between the empirical CKM matrices (11) and (2).

To study further the details of the various elements, it is convenient to consider the limit when the locations on the trajectory of the 2 highest generations are close together so as to make use of some familiar formulae in elementary differential geometry. This is seen in Figure 3 to be a reasonable approximation at least for the 3 fermion-types $U, D, L$. In this case, the triad of state vectors in Figure 1 becomes the so-called Darboux triad [16] with (i) $\mathbf{v}_{\mathbf{3}}$ being the (radial) vector normal to the surface (sphere), (ii) $\mathbf{v}_{\mathbf{2}}$ the tangent vector to the curve (trajectory), and (iii) $\mathbf{v}_{\mathbf{1}}$ the vector orthogonal to both. And the CKM matrix becomes just the rotation matrix of the Darboux triad on transporting it along the trajectory from the $U$ to the $D$ location for quarks, or from the $L$ to the $N$ location for leptons. To first order in the displacment, this rotation matrix is given by a variant of the well-known Serret-Frenet formula:

$$
C K M \sim\left(\begin{array}{ccc}
1 & -\kappa_{g} \Delta s & -\tau_{g} \Delta s \\
\kappa_{g} \Delta s & 1 & \kappa_{n} \Delta s \\
\tau_{g} \Delta s & -\kappa_{n} \Delta s & 1
\end{array}\right) .
$$

For our special case of a curve on a unit sphere the geodesic torsion vanishes $\tau_{g}=0$ and the normal curvature is constant $\kappa_{n}=1$. As a result, one concludes immediately (b) that the corner elements of the CKM matrix, being of at least second order in the the displacement $\Delta s$, are much smaller than the others, and (c) that the 23 and 32 elements, being proportional to the separation between $t$ and $b$ for quarks and between $\tau$ and $\nu_{3}$ for leptons, are much smaller for the quark than for the lepton CKM matrix. Again, as already noted at the beginning, these predictions are strongly borne out by experiment. The other two off-diagonal elements depend on the geodesic curvature $\kappa_{g}$ which depends in turn on both the trajectory and the location on it, and will be harder for the present mechanism to predict.

One sees therefore that, even without performing any calculation, one is already able to explain qualitatively most of the outstanding features in the mixing pattern and the hierarchical mass spectrum of both quarks and 
leptons. What remains now is to attempt an actual fit with our 3 parameters and see if one gets reasonable quantitative agreement. We propose to proceed as follows. Of the quantities we can calculate, the most accurately measured experimentally are the 2 mass ratios $m_{c} / m_{t}, m_{\mu} / m_{\tau}$ and the Cabibbo angle $V_{c d} \sim V_{u s}$. We shall therefore determine our 3 parameters by fitting the experimental values of these 3 quantities. Having then decided on a trajectory for the (normalized) vector $\left(x^{\prime}, y^{\prime}, z^{\prime}\right)$ as encoded in some intitial value $\left(x_{I}, y_{I}, z_{I}\right)$, and on the value of the Yukawa coupling strength $\rho$ which governs the speed with which the vector runs along the trajectory, we can then just follow the procedure given above to calculate the other parameters. We have to input the (Dirac) masses of the heaviest generation. For the $U$ - and $D$-type quarks and charged leptons, we take from [1]:

$$
m_{t}=173.8 \mathrm{GeV}, \quad m_{b}=4.247 \mathrm{GeV}, \quad m_{\tau}=1.777 \mathrm{GeV},
$$

the chosen value for $m_{b}$ being the geometric mean of the given experimental limits. With these inputs, we calculate the masses of $c$ and $\mu$ and the quark CKM matrix elements $V_{u s}$ and $V_{c d}$, adjusting the values of the Yukawa coupling strength $\rho$ and the initial values of the vector $\left(x_{I}, y_{I}, z_{I}\right)$ until we obtain the experimental values given in [1], namely:

$$
m_{c}=1.1-1.4 \mathrm{GeV}, \quad m_{\mu}=105.6 \mathrm{MeV}, \quad V_{u s}, V_{c d}=0.217-0.224 .
$$

This requires running the vector $\left(x^{\prime}, y^{\prime}, z^{\prime}\right)$ numerically with the formula (12) from the initial value $\left(x_{I}, y_{I}, z_{I}\right)$ down to the second heaviest generation for each fermion-type. We take typically around 500 steps for each decade of energy to achieve about 1 percent accuracy, normalizing the vector $\left(x^{\prime}, y^{\prime}, z^{\prime}\right)$ again at every step. The quantities $\rho$ and $m_{T}$ which in principle also run are taken here, for lack of anything better, to be constants, any slow variations of which, we believe, would be masked in practice by adjustments of the free parameters to fit the values in (15). With the values of $\rho$ and $x_{I}, y_{I}, z_{I}$ so obtained, we can then make predictions for other quantities.

We distinguish two categories of such predictions. The first requires only the running between the heaviest and second heaviest generations which category is expected to be more reliable given that our parameters have been determined from running in the same range. These predictions include all the CKM matrix elements for both quarks and leptons, and the masses of the strange quark $m_{s}$ and the 'right-handed neutrino' $B$. A list of such predictions on the CKM matrix elements is given in Table 11 where the 'predicted 
central value' is obtained by putting $m_{t}=173.8 \mathrm{GeV}$, the experimental central value, $m_{c}=1.241 \mathrm{GeV}$, the geometric mean of the experimental limits, and $\frac{1}{2}\left(V_{u s}+V_{c d}\right)=0.2205$, the (arithmetic) mean of the experimental limits, giving for the 'central values' of the fitted parameters:

$$
\rho=3.535, \quad x_{I}=0.9999984, y_{I}=0.0017900, z_{I}=0.0000179,
$$

where the initial value of the vector $\left(x_{I}, y_{I}, z_{I}\right)$ is taken arbitrarily at the scale of $20 \mathrm{TeV}$. The 'predicted range' is obtained by varying $m_{t}$ within the quoted experimental error of $\pm 5.2 \mathrm{GeV}$, and $m_{c}$ and $V_{u s}, V_{c d}$ within their experimental limits quoted in (15) above, and corresponds to the range of the fitted parameters:

$$
\begin{array}{ll}
\rho=3.393-3.688, & x_{I}=0.9999959-0.9999994, \\
y_{I}=0.0010800-0.0028500, & z_{I}=0.0000075-0.0000391 .
\end{array}
$$

The agreement between prediction and experiment for the quark CKM matrix in Table 1 is seen to be good for all entries.

For neutrinos, as explained above, we need to input the physical masses of the two heaviest generations. Taking these as:

$$
m_{\nu_{3}}^{2}=3.5 \times 10^{-3} \mathrm{eV}^{2}, \quad m_{\nu_{2}}^{2}=4.3 \times 10^{-10} \mathrm{eV}^{2},
$$

which are the best fit values to the latest SuperKamiokande data given in [5, 7], one obtains the entries for the lepton CKM matrix in Table 1. On the other hand, if one varies these input masses within the range permitted still either by [2, 4] or by [3, 5, 7]:

$$
m_{\nu_{3}}^{2}=(1.2-30) \times 10^{-3} \mathrm{eV}^{2}, \quad m_{\nu_{2}}^{2}=(0.6-7.9) \times 10^{-10} \mathrm{eV}^{2},
$$

while keeping the central values (16) of the fitted parameters, one obtains:

$$
U_{\mu 3}=0.6434-0.7108, \quad U_{e 3}=0.0617-0.0814, \quad U_{e 2}=0.2221-0.2352 \text {. }
$$

The agreement with experiment is again seen consistently to be good, except for $U_{e 2}$. Notice in particular, by comparing with the quark matrix, the close agreement with the outstanding features (a)-(c) of the empirical mixing matrices noted at the beginning. The element $U_{e 3}$ is small as required by [6] while $U_{\mu 3}$ responsible for the muon anomaly in atmospheric neutrinos 
is near maximal corresponding to $\sin ^{2} 2 \theta>0.97$. As for $U_{e 2}$, the mixing angle involved in oscillations of solar neutrinos, we recall from (13) above that, of the mixing elements in the CKM matrix, this element corresponding to the 'geodesic curvature' $\kappa_{g}$ is the one most sensitive to details in the present scheme, being dependent both on the trajectory and on the location on it. It is therefore not surprising that, though still of a reasonable order of magnitude, it does not come out as well as the others.

In addition, one predicts:

$$
m_{s}=173 \pm 5 \mathrm{MeV}, \quad B=300(223-418) \mathrm{TeV} .
$$

The value of $m_{s}$ given is the running mass taken at the scale equal to its value and cannot be directly compared with the values given in the data tables, e.g. $100-300 \mathrm{MeV}$ taken at $1 \mathrm{GeV}$ [17] or $70-170 \mathrm{MeV}$ taken at $2 \mathrm{GeV}$ [1], but is seen to be reasonable. The predicted value for $B$, which is of course experimentally yet unknown, is interesting in that it is much lower than usual GUT estimates and leads to much more accessible rates for neutrinoless double beta decays, only $2-3$ orders of magnitude lower than the present limit.

The other category of predictions requires running further down in energy scale down to the lightest generation with parameters fixed by fitting the two heavier generations. First, being extrapolations on a logarithmic scale, they are in any case not expected to be reliable except as rough order-ofmagnitude estimates. Secondly, for quarks, nonperturbative QCD corrections are important below 1-2 GeV, which are hard to estimate. Nevertheless if one persists, assuming still $\rho$ and $m_{T}$ to be constants, one obtains:

$$
m_{u}=200 \mathrm{MeV}, \quad m_{d}=15 \mathrm{MeV}, \quad m_{e}=6 \mathrm{MeV}, \quad m_{\nu_{1}} \sim 2 \times 10^{-15} \mathrm{eV},
$$

to be compared with the experimental numbers:

$$
m_{u}=1.5-5 \mathrm{MeV}, \quad m_{d}=3-9 \mathrm{MeV}, \quad m_{e}=0.51 \mathrm{MeV}, \quad m_{\nu_{1}}<10 \mathrm{eV} .
$$

While $m_{d}$ and $m_{e}$ may be considered reasonable given the expected inaccuracy and $m_{\nu_{1}}$ has of course no difficulty in satisfying the experimental bound, the predicted value for $m_{u}$ is some 2 orders out. It should be stressed, however, that the predicted value for $m_{u}$ is defined as the running mass taken at the scale equal to its value, and it is unclear whether it should be compared 


\begin{tabular}{||c||c||c|c||}
\hline \hline Quantity & ExperimentalRange & $\begin{array}{c}\text { Predicted } \\
\text { CentralValue }\end{array}$ & PredictedRange \\
\hline \hline$\left|V_{u d}\right|$ & $0.9745-0.9760$ & 0.9753 & $0.9745-0.9762$ \\
\hline$\left|V_{u s}\right|$ & $0.217-0.224$ & $(0.2207)$ & \\
\hline$\left|V_{u b}\right|$ & $0.0018-0.0045$ & 0.0045 & $0.0043-0.0046$ \\
\hline$\left|V_{c d}\right|$ & $0.217-0.224$ & $(0.2204)$ & \\
\hline$\left|V_{c s}\right|$ & $0.9737-0.9753$ & 0.9745 & $0.9733-0.9756$ \\
\hline$\left|V_{c b}\right|$ & $0.036-0.042$ & 0.0426 & $0.0354-0.0508$ \\
\hline$\left|V_{t d}\right|$ & $0.004-0.013$ & 0.0138 & $0.0120-0.0157$ \\
\hline$\left|V_{t s}\right|$ & $0.035-0.042$ & 0.0406 & $0.0336-0.0486$ \\
\hline$\left|V_{t b}\right|$ & $0.9991-0.9994$ & 0.9991 & $0.9988-0.9994$ \\
\hline$\left|V_{u b} / V_{c b}\right|$ & $0.08 \pm 0.02$ & 0.1049 & $0.0859-0.1266$ \\
\hline$\left|V_{t d} / V_{t s}\right|$ & $<0.27$ & 0.3391 & $0.3149-0.3668$ \\
\hline$\left|V_{t b}^{*} V_{t d}\right|$ & $0.0084 \pm 0.0018$ & 0.0138 & $0.0120-0.0156$ \\
\hline \hline$\left|U_{\mu 3}\right|$ & $0.56-0.83$ & 0.6658 & $0.6528-0.6770$ \\
\hline$\left|U_{e 3}\right|$ & $0.00-0.15$ & 0.0678 & $0.0632-0.0730$ \\
\hline$\left|U_{e 2}\right|$ & $0.4-0.7$ & 0.2266 & $0.2042-0.2531$ \\
\hline \hline
\end{tabular}

Table 1: Predicted CKM matrix elements for both quarks and leptons 
with the quoted experimental value defined at the scale of $2 \mathrm{GeV}$. Indeed, if one simply calculates the expectation value in the $u$-state of the running mass matrix $m^{\prime}$ at $\mathrm{GeV}$ scale, one obtains a value of order only $1 \mathrm{MeV}$, but it is also unclear whether this is the number to be compared to the quoted experimental value. Barring this ambiguity, which applies also to $m_{d}$, the comparison to experiment at an order-of-magnitude level is not unreasonable as the masses do at least follow the clear hierarchical pattern seen in experiment.

One concludes therefore that simply by assuming that generations originate in an $S U(3)$ gauge symmetry broken in the particular manner of (3), one can already explain the main empirical features in the mixing pattern together with the hierarchical mass spectrum of the Standard Model fermions. An important feature of the mechanism is that the mixing pattern and the hierarchical mass spectrum are intimately related. In particular, one recalls that for neutrinos, the mass ratio $m_{\nu_{2}} / m_{\nu_{3}}$ between the two heaviest generations cannot be as large as that required by the standard MSW solutions to the solar neutrino problem, or otherwise one finds no solution with the present mechanism, which admits only mass ratios of the order of that required by the vacuum or long wave-length (LWO) solution. Hence, if the preference of the recent SuperKamiokande data for the (LWO) solution (iii) is maintained, it would lend support to this mechanism.

Further, one has recovered here the bulk of the phenomenological output of what we called the Dualized Standard Model (DSM) without having introduced at all the concept of nonabelian duality on which that scheme is based [10]. The only phenomenological consequence of DSM so far studied which has been missed by the considerations here is the possible explanation of cosmic ray air showers beyond the Greisen-Zatsepin-Kuz'min cut-off. There seems thus a valid case to consider the present mechanism on its own independently of the original 'dual' tenets of the DSM. Indeed, one might attempt to go a step further and bypass even the particular symmetry breaking scheme embodied in the Higgs potential (3), for the main effect of that was really just to make the mass matrix factorize and rotate with respect to the energy scale. If one can devise some other scheme in which a similar situation attains, then an analogous conclusion is likely to be achievable for explaining the empirically observed mixing pattern.

We ourselves, however, adhere to our preference for the original DSM scheme. The reason is that not only does the dynamical mechanism examined 
in this paper arise naturally there as a consequence of the dual framework, but even the very existence itself of a broken $S U(3)$ gauge symmetry and of the Higgs fields required for its breaking emerges automatically from the concept of nonabelian duality. Indeed, if one accepts this concept, then the niches for 'generations' and 'Higgs fields' would in any case already exist in the Standard Model, and if they are not assign these their seemingly natural physical roles, they would still have to be accounted for in some other manner, which may not be easy to come by.

Lastly, it should be stressed that although the main features of fermion mass and mixing patterns are shown to follow from the dynamical mechanism described in this paper, no consideration has been given here for possibly other predictions of the same mechanism violating experiment. For the DSM scheme, some considerations have been given to these questions, but if this mechanism is grafted on to some other specific scheme, such questions will of course have to be readdressed.

\section{Acknowledgement}

One of us (JB) is supported in part by grants CYCIT96-1718, PB971261 and GV98-1-80. He would also like to thank the Rutherford Appleton Laboratory for hospitality.

\section{References}

[1] Particle Physics Booklet, (1998), from C. Caso et al., The European Physical Journal C3, 1, (1998).

[2] K.S. Hirata et al., Phys. Letters, B205, 416, (1988); B280, 146, (1992); Y. Fukuda et al. Phys. Letter B335, 237, (1994).

[3] SuperKamiokande data, in talks presented by C. McGrew and M. Vagins at ICHEP'98, Vancouver (1998).

[4] Soudan II data, in talk presented by H. Gallagher at ICHEP'98, Vancouver (1998).

[5] Talk given by Mark Messier at APS meeting (DPF) at UCLA, Los Angeles, Jan. 5-9, 1999, http://www.physics.ucla.edu/dpf99/trans. 
[6] CHOOZ collaboration, M. Apollonio et al., Phys. Lett. B420, 397, (1997).

[7] Talk given by Michael B. Smy at APS meeting (DPF) at UCLA, Los Angeles, Jan. 5-9, 1999, http://www.physics.ucla.edu/dpf99/trans.

[8] Chan Hong-Mo, José Bordes, and Tsou Sheung Tsun, hep/ph-9809272, Rutherford Laboratory preprint RAL-TR-98-071, talk given at the Vancouver ICHEP'98 conference, to appear in the proceedings.

[9] Chan Hong-Mo and Tsou Sheung Tsun, hep-th/9701120, Phys. Rev. D57, 2507, (1998).

[10] Chan Hong-Mo, J. Faridani and Tsou Sheung Tsun, Phys. Rev. D53, 7293, (1996).

[11] see e.g. [9].

[12] José Bordes, Chan Hong-Mo, Jacqueline Faridani, Jakov Pfaudler, and Tsou Sheung Tsun, hep-ph/9712276, Phys. Rev. D58, 013004, (1998)

[13] José Bordes, Chan Hong-Mo, Jacqueline Faridani, Jakov Pfaudler, and Tsou Sheung Tsun, hep-ph/9807277, Valencia preprint (1998).

[14] For an example of a recent analysis, see G.L. Fogli, E. Lisi, and D. Montanino, Phys. Rev. D54, 2048 (1995).

[15] V. Barger, R.J.N. Phillips, and K. Whisnant, Phys. Rev. Letters, 69, 3135, (1992); P.I. Krastev and S.T. Petcov, Phys. Rev. Letters, 72, 1960, (1994).

[16] See e.g. L.P. Eisenhart, A Treatise on the Differential Geometry of Curves and Surfaces, Ginn and Company 1909, Boston; M.P. do Carmo, Differential Geometry of Curves and Surfaces, Prentice-Hall 1976, Englewood Cliffs, New Jersey.

[17] Particle Physics Booklet, (1996), from R.M. Barnett et al., Phys. Rev. D54, 1, (1996). 